\title{
A reflective-backing-free metamaterial absorber with broadband response
}

\author{
Cuilian Xu, Shaobo Qu*, Jiafu Wang, Mingbao Yan, Yongqiang Pang, \\ Wenjie Wang and Hua Ma \\ Science College, Air Force Engineering University \\ Xi'an 710051, P. R. China \\ *qushaobo@mail.xjtu.edu.cn
}

Received 31 March 2017; Revised 20 April 2017; Accepted 30 April 2017; Published 19 June 2017

\begin{abstract}
In this paper, we propose a polarization-independent and broadband perfect infrared (IR) metamaterial absorber (MA) without reflective backing. The proposed absorber is a periodic meta-atom array consisting of metal-dielectric-multilayer truncated cones which can absorb $80 \%$ EM wave from 50.70 to $81.87 \mathrm{THz}$, while transmit $80 \%$ EM wave from 0 to $37.71 \mathrm{THz}$. With the decreasing of frequency, the transmissivity increases, which is close to $100 \%$ from 0 to $5 \mathrm{THz}$. We can broaden the absorption bandwidth of the MA by cascading multi-layers truncated cones. Furthermore, the proposed IR MA promises to be one desirable stealth material for radar-IR compatibility.
\end{abstract}

Keywords: Metamaterial absorber; reflective-backing-free; infrared-radar stealth-compatible.

\section{Introduction}

In recent years, there has been a growing and wide-spread interest in absorption materials due to their military and civil applications, such as stealth defense system, microwave interference protection, solar cells, and infrared selective thermal emitters. Metamaterial absorber (MA), which can obtain near unity or perfect absorption at a certain frequency with the help of structural design, have attracted considerable attention. Therefore, several types of perfect MAs are designed at specific wavelength ranging from microwave region ${ }^{1-3}$ to the optical wavelength, ${ }^{4,5}$ as well as in the infrared (IR) ${ }^{6-9}$ However, these absorbers are usually backed by a metallic plate. ${ }^{10-13}$ The usual function of metallic backing is to provide electric boundary condition for incident plane wave, and enables to create magnetic resonance. Besides, it is used to avoid power transmission on the other side of the absorber.

However, the presence of the metallic plate may encounter a problem, especially for stealth applications. The most important research in stealth material technology is aimed at compatible stealth functionality in IR and radar wave bands. In IR region, the absorbing materials require a lower emissivity while the materials demand a high absorptive and low reflectivity in radar wave range. The infrared MA with a metallic backing exhibit high reflectivity in radar waveband. It is very difficult to unify the infrared and radar stealth. Moreover, for radar absorbers, we need to hide the object at a given frequency, in fact, the employment of conventional absorbers with a metallic backing makes the radar crosssection (RCS) of the object increases outside its absorption band. Thus, it may affect stealth performance of the object severely. In addition, MA with a metallic backing is impossible to open EM-wave transmission window at frequencies outside its absorption band. The EM-wave transmission window is protecting equipment of antenna system which is installed on radar, guidance, and communication systems.

Therefore, it is desirable and necessarily to design reflective-backing free MA. Filiberto Bilotti proposed a design of microwave absorbers without a metallic backing based on magnetic inclusions behaviors and discussed its advantage in stealth. ${ }^{14}$ The physical mechanism is similar to the Salisbury screen, ${ }^{15}$ a $377 \Omega$ resistive sheet is also used to absorb the electromagnetic energy of the impinging field. Moreover, the absorber ${ }^{16}$ proposed by Landy, which consists of the split-ring resonator (SRR) and metal wire, is also not backed by a metallic plate. However, these MAs without back metallic plane only have perfect absorption at a certainty frequency with a narrow bandwidth. So it cannot cover a single- or multi-band of radar wave. Qu shao-bo and Lu lei simulate and experimentally validate a polarization-independent transmission absorption MA, ${ }^{17}$ which can absorb the high-frequency electromagnetic wave, and transmit the lowfrequency wave. To further broaden the absorption bandwidth of the resonant MA, they place two absorbers with different working frequencies together for its low-frequency transmitted characteristic, but these three-dimensional absorbers are difficult to fabricate in the infrared region. In this study, we propose a polarization-independent and broadband perfect IR MA without back metallic plane, which can be used to construct the compatible stealth composite material of radar and IR. It is also promising for frequency selective absorbers (such absorbers with windows). ${ }^{18}$

This is an Open Access article published by World Scientific Publishing Company. It is distributed under the terms of the Creative Commons Attribution 4.0 (CC-BY) License. Further distribution of this work is permitted, provided the original work is properly cited. 


\section{Design of Metamaterial Absorber}

The schematic of the designed MA is presented in Fig. 1. It consists of alternating metallic and dielectric thin films. The parameters of the unit cell are marked in Fig. 1. The periodicity of the unit cell is $P=2.5 \mu \mathrm{m}$, the diameters of metallic circular discs from top to bottom are $D_{1}=1.4 \mu \mathrm{m}$, $D_{2}=1.6 \mu \mathrm{m}, D_{3}=1.8 \mu \mathrm{m}$, and $D_{4}=2.0 \mu \mathrm{m}$, respectively. The employed metallic layer was the Aluminium (Al), and dielectric layer was Alumina $\left(\mathrm{Al}_{2} \mathrm{O}_{3}\right)$, the thicknesses of the Al layer and $\mathrm{Al}_{2} \mathrm{O}_{3}$ layer are $t_{m}=0.04 \mu \mathrm{m}$ and $t_{d}=0.07 \mu \mathrm{m}$, respectively.

Numerical calculations were performed by finite difference time domain (FDTD) method in CST Microwave Studio. Aluminium was characterized by a Drude model with a plasma frequency $\omega_{p}=2 \pi \times 3.57 \times 10^{15} \mathrm{rad} / \mathrm{s}$ and collision frequency $\omega_{\gamma}=2 \pi \times 19.41 \times 10^{12} \mathrm{rad} / \mathrm{s}$. Alumina $\left(\mathrm{Al}_{2} \mathrm{O}_{3}\right)$ was modeled with dielectric constant of 2.28 and loss tangent of 0.04. All materials are assumed to be nonmagnetic (i.e., $\left.\mu=\mu_{0}\right)$. Periodic boundary conditions are used for both $x$ - and $y$-directions, and a open (add space) boundary condition was employed in the $z$-direction. The absorption is given by $A(\omega)=1-R(\omega)-T(\omega)=1-\left|S_{11}(\omega)\right|^{2}-\left|S_{21}(\omega)\right|^{2}$. $A(\omega), R(\omega)$ and $T(\omega)$ are absorptance, reflectance and transmittance, respectively. $S_{11}(\omega)$ and $S_{21}(\omega)$ are the scattering parameters of reflection and transmission, respectively. A normal incident plane wave was applied with the polarization along with the $x$-direction.

\section{Numerical Analysis and Discussion}

The reflection, absorption and transmission spectrum of the multiband absorber are shown in Fig. 2(a). We can clearly see that three absorption peaks occur and locate at 54.6, 61.2, and $69.8 \mathrm{THz}$, respectively, each peak exceeds $80 \%$. The near unity absorption of the structure results from the coupling of the electric and magnetic resonances. In low frequency, the reflection is low while the transmission is high, so most power of incident EM-wave transmit through the other side of the absorber. The curve of absorption and transmission spectrum intersect at $69.8 \mathrm{THz}$ with the increasing of frequency, here the value of reflectivity and the transmissivity are all small, we obtained the first absorption peaks. In high frequency, the reflectivity is high while the transmissivity is low, most power of incident EM-wave were reflected. Furthermore, we can broaden absorption bandwidth making use of the high transmissivity characteristic in low-frequency regime. Through increasing layers of metal and dielectric and changing the parameters of the structure. We designed a

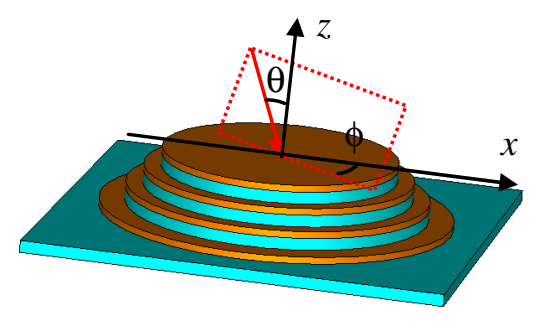

(a)

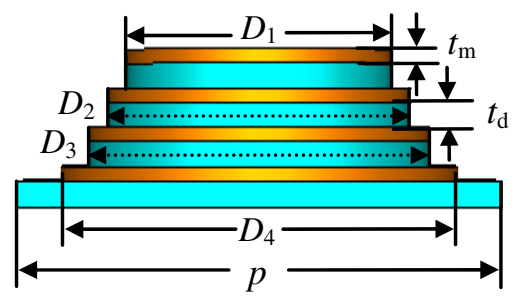

(b)

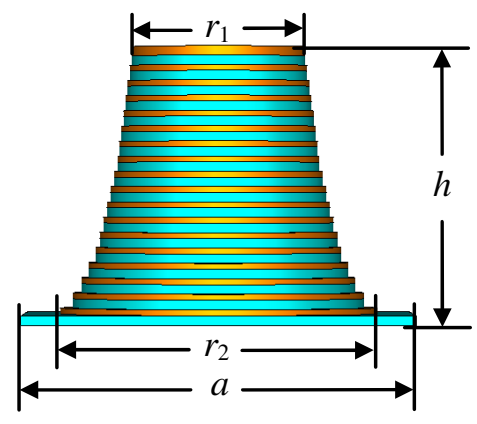

(c)

Fig. 1. (a) Schematic diagram and (b) side view of the proposed multiband MA; (c) the construction of the broad-band MA.

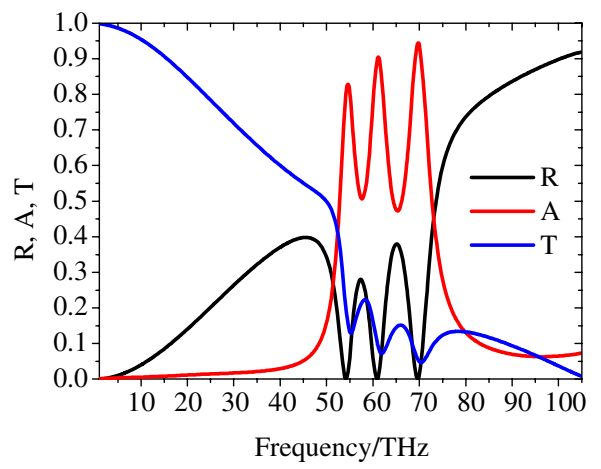

(a)

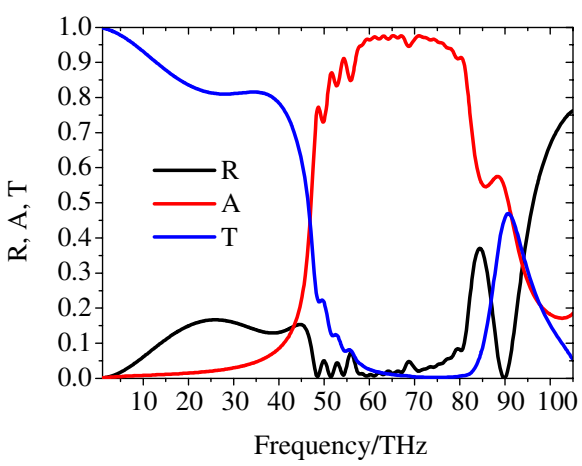

(b)

Fig. 2. Reflection, absorption and transmission spectrum of the MA (a) multiband absorber; (b) broad-band absorber. 
polarization-independent and broad-band MA without metallic backing operating in mid-IR regime as schematically depicted in Fig. 1(c), the periodicity of the unit cell is a $=2.7 \mu \mathrm{m}$, the widths of top and bottom circular discs are $r_{1}=2.2 \mu \mathrm{m}$ and $r_{2}=1.2 \mu \mathrm{m}$, the total number of metal/ dielectric pairs $(N)$ is 18 , the total thickness is $h=1.98 \mu \mathrm{m}$. The proposed broadband MA which can absorb $80 \%$ EM wave from 50.70 to $81.87 \mathrm{THz}$, while transmitting $80 \% \mathrm{EM}$ wave from 0 to $38.71 \mathrm{THz}$, is shown in Fig. 2(b). It is worth noticing that with the decreasing of frequency, the transmissivity increases, which is close to $100 \%$ from 0 to $5 \mathrm{THz}$.

Moreover, due to the symmetrical characteristic of truncated cones structure, it has almost the same response to transverse electric (TE) polarized light and transverse magnetic (TM) polarized light for normal incidence (as shown in Fig. 2). In Fig. 3, we investigate the stability of multiband and broadband absorptions with different azimuthal angles $\varphi$ at $\theta=0^{\circ}$. It can be seen that the intensity of the two absorption peaks is stable varying with different azimuthal angles from $0^{\circ}$ to $85^{\circ}$. In Fig. 4, we investigate the stability of transmission with different azimuthal angles $\varphi$ at $\theta=0^{\circ}$, the transmission is polarization-independent, and the transmissivity all exceed $90 \%$ varying with different azimuthal angles from 0 to $12.76 \mathrm{THz}$. Moreover, due to the effects of multiple metal/dielectric thin films and the truncated cone shapes, the proposed metamaterial structure can greatly suppress the reflection of light by effectively providing a graded transition of refractive index, and thus acts as an ultra-broadband

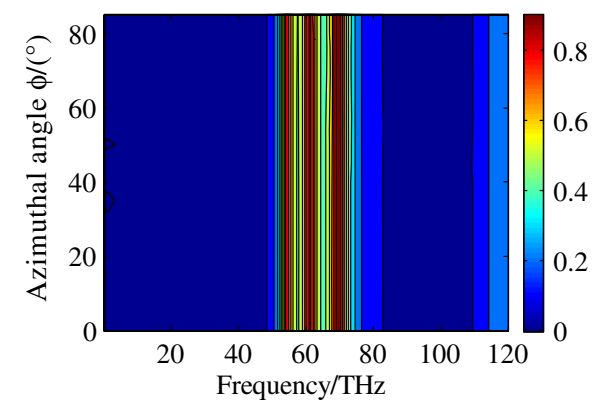

(a)

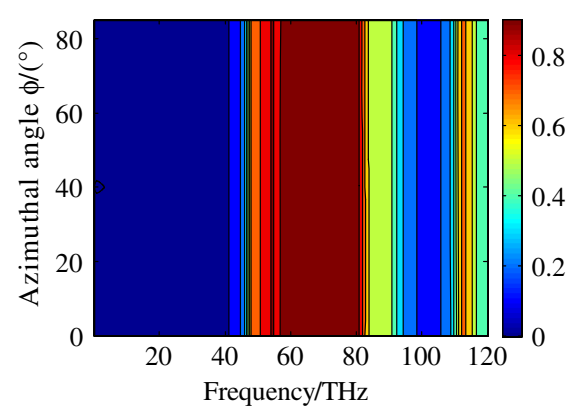

(b)

Fig. 3. Absorption spectrum as a function of azimuthal angle $\varphi$ at $\theta=0^{\circ}$ for TM polarization (a) for multiband absorber and (b) for broadband absorber.

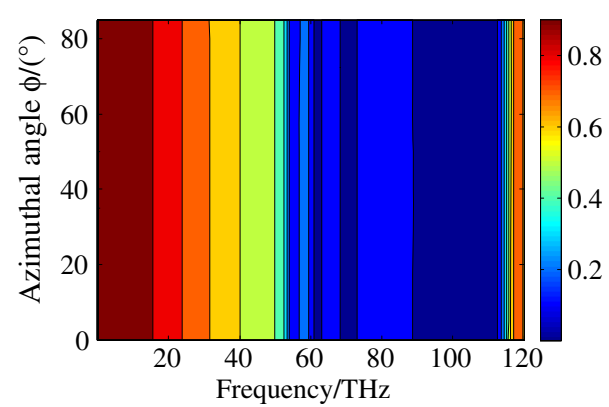

(a)

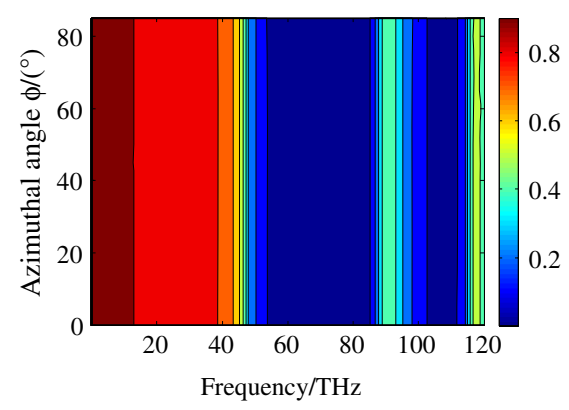

(b)

Fig. 4. Comparison of the monochromatic radiance exitance for blackbody, low emissivity coating and the proposed FSTE (a) $T=445 \mathrm{~K}$; (b) $T=600 \mathrm{~K}$.

multilayer antireflection coating as well. ${ }^{19}$ Thus, the present simulation results demonstrate that the broadband absorber structure have excellent transmissivity in low-frequency.

To better understand the mechanism of the resonant absorption behavior, we monitored the electric and magnetic field distributions at three different frequencies for the TM polarized incident wave. The results are illustrated in Fig. 5 for $f=69.8,61.2$ and $54.6 \mathrm{THz}$, respectively. Figure 5 shows that enhancement of the electric-magnetic field has been excited at specific part of the multilayer absorber for the specific wavelength, the positions of the induced electric and
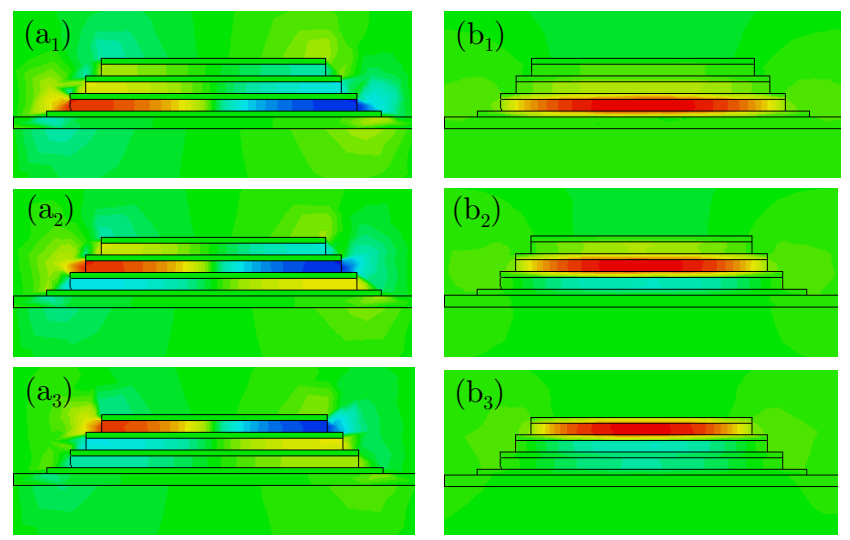

Fig. 5. Distributions of $z$-component electric field (real $\left(E_{z}\right)$ ) $\left(\mathrm{a}_{1}-\mathrm{a}_{3}\right)$ and the $y$-component magnetic field $\left(H_{y}\right)\left(\mathrm{b}_{1}-\mathrm{b}_{3}\right)$ in the plane $y=0$ of unit cell at $54.6,61.2$, and $69.8 \mathrm{THz}$. 


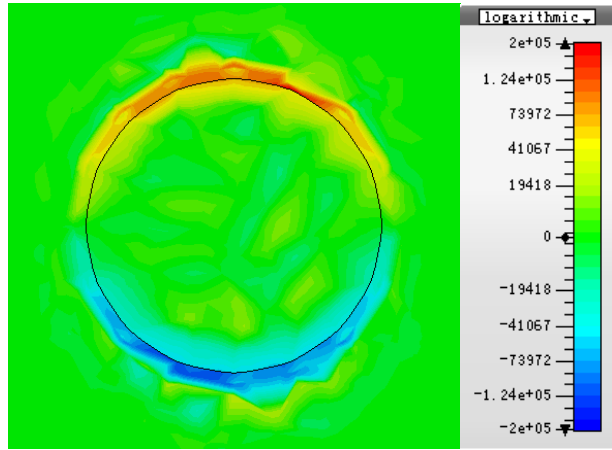

(a)

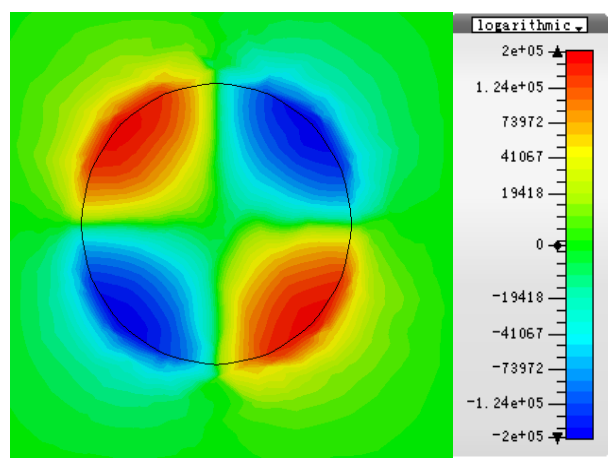

(b)

Fig. 6. Distributions of (a) the $z$-component electric field (real $\left(E_{z}\right)$ ); (b) the $y$-component electric field (real $\left.\left(E_{y}\right)\right)$ at the metal/dielectric interface $(z=0.297 \mu \mathrm{m}$ plane), when the frequency $f=69.8 \mathrm{THz}$.

magnetic fields are shifted continuously to the top of the MA structures with the increasing of frequency. Such as at $54.6 \mathrm{THz}$, the induced electric field and the induced magnetic field are located in the bottom of the structures. To obtain a physical insight, distributions of the $z$-component electric field (real $\left.\left(E_{z}\right)\right)$ and the $y$-component electric field (real $\left(E_{y}\right)$ ) at the metal/dielectric interface are shown in Fig. 6 (here we choose the plane $z=0.297 \mu \mathrm{m}$ with wavelength $f=$ $69.8 \mathrm{THz}$, as an example). As shown in Figs. $5\left(\mathrm{a}_{1}\right)-5\left(\mathrm{a}_{3}\right)$, Figs. 6(a) and 6(b), opposite charges accumulate at the edges of the metal films, which indicates the excitation of electric dipole resonance on the metal films. Moreover, the electric dipoles are greatly coupled with their own images, and oscillate in antiphase on neighboring metallic. We can see from Figs. $5\left(a_{2}\right)$ and $5\left(a_{3}\right)$ that the electric field profile in neighboring dielectric are just opposite in sign compared with that on the topper patch. Consequently, magnetic polaritons are formed, which induce strong magnetic response and cause strong enhancement of the magnetic field. Therefore, the absorption of multilayered structure is formed not only by the electric resonance but also by the magnetic resonance. ${ }^{19}$ Figure 6(b) shows the $y$-component electric field at the metal/ dielectric interface, it can be seen that the magnetic field and electric field were concentrated in dielectric layers obviously, the strong absorption mostly root in dielectric loss of dielectric layers.

It should be mentioned that the induced magnetic field is located at the center while the induced electric field is formed at the sides. That is because the induced surface current only flows through metal film, naturally the induced electric field is strong at the sides of dielectric layer in terms of equivalent oscillating-current resonant circuit. However, the magnetic field is induced by the antiparallel surface current at two neighboring layers, so the magnetic field is located at the center. $^{20}$

\section{Conclusion}

We propose a polarization-independent multiband MA without metallic backing operating in mid-IR regime. The simulated results show that the proposed multiband absorber has three absorption peaks located at 54.6, 61.2, and $69.8 \mathrm{THz}$, each with the absorption over $80 \%$. We can broaden the absorption bandwidth of the MA by cascading multi-layers truncated cones, and obtained a broadband absorber which can absorb $80 \%$ EM wave from 50.70 to $81.87 \mathrm{THz}$, while transmitting $80 \%$ EM wave from 0 to $37.71 \mathrm{THz}$. Moreover, Combining this structure with RAM, compatible stealth of radar and infrared can be achieved. By adjusting the parameters of the truncated cones structure, the proposed MA can obtain a high absorptivity performance, not only at IR frequencies but also at microwave band. The MA without metallic backing operating in microwave region can depress the RCS of the object outside its absorption band. It can also be used to communicate straightway in low frequency and shield security of information in high frequency, so it is possible to open EM-wave transmission window at frequencies outside its RAM absorption band.

\section{Acknowledgments}

The work was supported by the National Science Foundation of China (Grant Nos. 61331005, 60501502 and 61501497).

\section{References}

${ }^{1}$ G. Z. Wang and B. X. Wang, Five band terahertz metamaterial absorber based on a four gap comb resonator, J. Lightwave Technol. 33, 24 (2015).

${ }^{2}$ Y. Q. Xu, P. H. Zhou, H. B. Zhang, L. Chen and L. J. Deng, A wide-angle planar metamaterial absorber based on split ring resonator coupling, J. Appl. Phys. 110, 4 (2011).

${ }^{3}$ F. Ding, Y. Cui, X. Ge, Y. Jin and S. He, Ultra-broadband microwave metamaterial absorber, Appl. Phys. Lett. 100, 10 (2012).

${ }^{4} \mathrm{C}$. Hu, Z. Zhao, X. Chen and X. Luo, Realizing near-perfect absorption at visible frequencies, Opt. Express 17, 13 (2009).

5 J. Hao, J. Wang, X. Liu, W. J. Padilla, L. Zhou and M. Qiu, High performance optical absorber based on a plasmonic metamaterial, Appl. Phys. Lett. 96, 25 (2010). 
${ }^{6}$ N. Liu, M. Mesch, T. Weiss, M. Hentschel and H. Giessen, Infrared perfect absorber and its application as plasmonic sensor, Nano Lett. 10, 7 (2010).

${ }^{7}$ X. Liu, T. Tyler, T. Starr, A. F. Starr, N. M. Jokerst and W. J. Padilla, Taming the blackbody with infrared metamaterials as selective thermal emitters, Phys. Rev. Lett. 107, 4 (2011).

${ }^{8}$ M. Diem, T. Koschny and C. M. Soukoulis, Wide-angle perfect absorber/thermal emitter in the terahertz regime, Phys. Rev. B 79, 3 (2009).

${ }^{9}$ Y. Cui, K. H. Fung, J. Xu, H. Ma, Y. Jin, S. He and N. X. Fang, Ultrabroadband light absorption by a sawtooth anisotropic metamaterial slab, Nano Lett. 12, 3 (2012).

${ }^{10}$ W. Ma, Y. Z. H. Wen and X. M. Yu, Broadband metamaterial absorber at mid-infrared using multiplexed cross resonators, Opt. Express 21, 25 (2013).

${ }^{11}$ Y. Bai, L. Zhao, D. Q. Ju, Y. Y Jiang and L. H. Liu, Wide-angle, polarization-independent and dual-band infrared perfect absorber based on L-shaped metamaterial, Opt. Express 23, 7 (2015).

${ }^{12}$ Y. Li, B. An, S. Jiang, J. Gao, Y. Chen and S. Pan, Plasmonic induced triple-band absorber for sensor application, Opt. Express 23, 13 (2015).

${ }^{13}$ G. Z. H. Wang and B. X. Wang, Five-band terahertz metamaterial absorber based on a four-gap comb resonator, J. Lightwave Technol. 33, 24 (2015).
${ }^{14}$ F. Bilotti, A. Toscano, K. B. Alici, E. Ozbay and L. Vegni, Design of miniaturized narrowband absorber based on resonant-magnetic inclusions, IEEE Trans. Electromagn. Compat. 53, 1 (2011).

${ }^{15}$ R. L. Fante and M. T. McCormack, Reflection properties of the Salisbury screen, IEEE Trans. Antennas Propag. 30, 10 (1968).

${ }^{16}$ N. I. Landy, S. Sajuyigbe, J. J. Mock, D. R. Smith and W. J. Padilla, Perfect metamaterial absorber, Phys. Rev. Lett. 100, 20 (2008).

${ }^{17}$ L. Lu, S. B. Qu, H. Ma, F. Yu, S. Xia, Z. Xu and P. Bai, A polarization-independent transmission absorption metamaterial absorber based on electromagnetic resonance, Acta Phys. Sin. 62, 10 (2013).

${ }^{18}$ Y. Shang, Z. Shen and S. Xiao, Frequency-selective rasorber based on square-loop and cross-dipole arrays, IEEE Trans. Antennas Propag. 62, 11 (2014).

${ }^{19}$ Q. Q. Liang, T. S. H. Wang, Z. W. Lu, Q. Sun, Y. Q. Fu and W. X. $\mathrm{Yu}$, Metamaterial based two dimensional plasmonic subwavelength structures offer the broadest waveband light harvesting, Adv. Opt. Mater. 1 (2013).

${ }^{20}$ Y. J. Kim, Y. J. Yoo, K. W. Kim, J. Y. Rhee, Y. H. Kim and Y. P. Lee, Dual broadband metamaterial absorber, Opt. Express 23, 4 (2015). 